\title{
IGR J16318-4848: 7 years of INTEGRAL observations
}

\section{Laura Barragán*; Jörn Wilms, Ingo Kreykenbohm, Manfred Hanke, Felix Fürst}

Dr. Karl Remeis-Sternwarte and ECAP, Sternwartstr. 7, 96049 Bamberg, Germany

E-mail: laura.barragan@sternwarte.uni-erlangen.de

\section{Katja Pottschmidt}

CRESST, University of Maryland Baltimore County, 1000 Hilltop Circle, Baltimore, MD 21250, USA

NASA Goddard Space Flight Center, ASD, Code 661, Greenbelt, MD 20771, USA

\section{Richard E. Rothschild}

Center for Astrophysics and Space Sciences, University of California, San Diego, 9500 Gilman Dr., La Jolla, CA 92093-0424, USA

\begin{abstract}
Since the discovery of IGR J16318-4848 in 2003 January, INTEGRAL has accumulated more than $5.8 \mathrm{Ms}$ in IBIS/ISGRI. We present the first extensive analysis of the archival INTEGRAL data (IBIS/ISGRI, and JEM-X when available) for this source, together with the observations carried out by XMM-Newton (twice in 2003, and twice in 2004) and Suzaku (2006).

The source is very variable in the long-term, with periods of low activity, where the source is almost not detected, and flares with a luminosity $\sim 10$ times greater than its average value $(5.4 \mathrm{cts} / \mathrm{s})$. IGR J16318-4848 is a HMXB containing a $\mathrm{sgB}[\mathrm{e}]$ star and a compact object (most probably a neutron star) deeply embedded in the stellar wind of the mass donor. The variability of the source (also in the short-term) can be ascribed to the wind of the optical star being very clumpy.

We study the variation of the spectral parameters in time scales of INTEGRAL revolutions. The photoelectric absorption is, with $N_{\mathrm{H}}$ around $10^{24} \mathrm{~cm}^{-2}$, unusually high. During brighter phases the strong K-alpha iron line known from XMM-Newton and Suzaku observations is also detectable with the JEM- $X$ instrument.
\end{abstract}

8th INTEGRAL Workshop "The Restless Gamma-ray Universe"- Integral2010,

September 27-30, 2010

Dublin Ireland

\footnotetext{
*Speaker.

†We acknowledge support from DLR grant 50OR0701.
} 


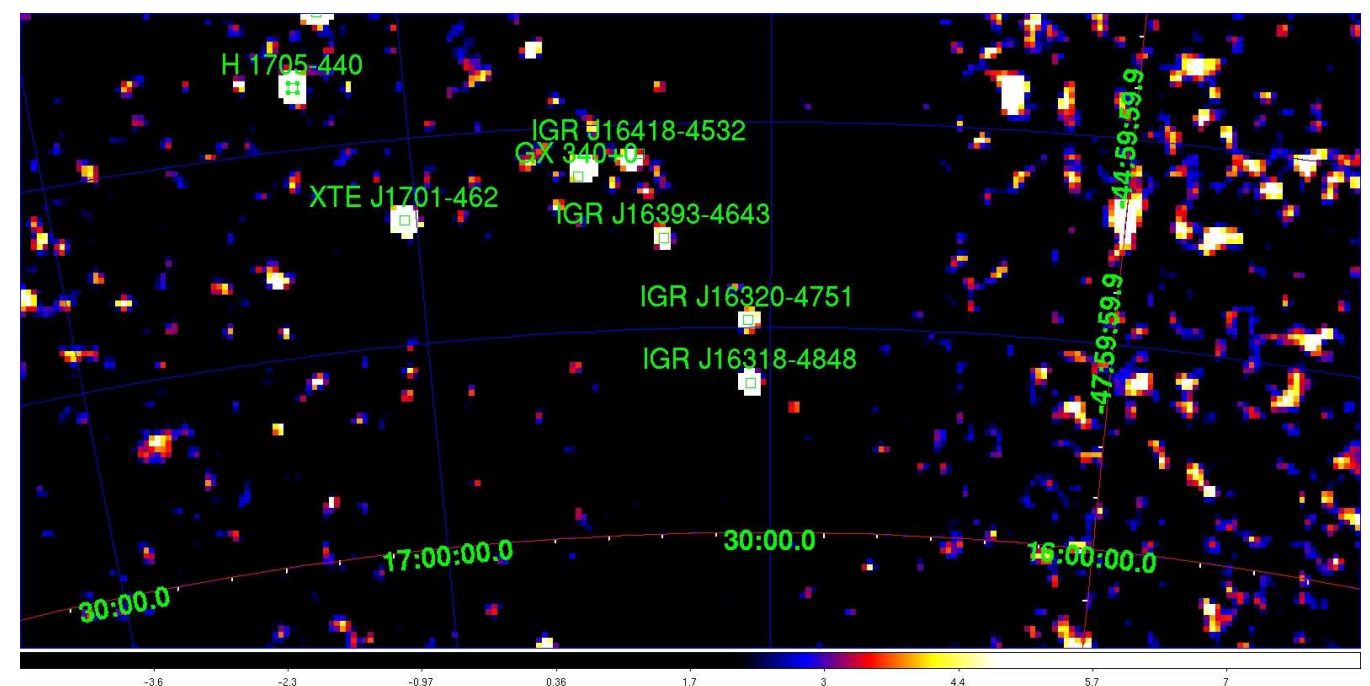

Figure 1: $29^{\circ} \times 9^{\circ}$ image obtained with IBIS/ISGRI in the $40-60 \mathrm{keV}$ range for the revolution 0468 .

\section{IGR J16318-4848}

IGR J16318-4848 was discovered on 2003 Jan 29 with the IBIS/ISGRI soft gamma-ray detector onboard INTEGRAL. It has been proposed that the source is a High Mass X-ray Binary (HMXB) with an $\operatorname{sgB}[\mathrm{e}]$ star as the mass donor (e.g., $[1,2,3]$ ) surrounded by a dense and absorbing circumstellar material. The distance is estimated to be between 0.9 and $6.2 \mathrm{kpc}$.

We analyzed the first Suzaku observation of IGR J16318-4848 [4], and described the average $\mathrm{X}$-ray spectrum of the source with a strongly absorbed power law continuum with a photon index of 0.676(42) and an exponential cutoff at 20.5(6) keV. These parameters are typical for accreting neutron stars (e.g., [5, 6, 3]). We searched for pulsations in the range between $1 \mathrm{~s}$ and $10 \mathrm{ksec}$ but could not find any coherent oscillation, so a black hole is not ruled out. Consistent with earlier work, strong fluorescent emission lines of $\mathrm{Fe} \mathrm{K} \alpha, \mathrm{Fe} \mathrm{K} \beta$, and $\mathrm{Ni} \mathrm{K} \alpha$ are observed, as well as a high column density, $N_{\mathrm{H}}=1.95(3) \times 10^{24} \mathrm{~cm}^{-2}$.

\section{Spectral Analysis}

Figure 2 shows the results of the analysis of the simultaneous data from IBIS/ISGRI and JEMX, including also the four XMM-Newton observations performed during 2003 and 2004. We fitted the same model we used for our Suzaku observation to the INTEGRAL data. The model consists of an absorbed cutoff powerlaw with three gaussians to account for the fluorescence lines, or only the ones for $\mathrm{Fe} \mathrm{K} \alpha$ and $\mathrm{K} \beta$ in the cases where we have only JEM-X as the low-energy instrument. Photoabsorption was modeled with a revised version of TBabs model ${ }^{1}$ [7], using the interstellar medium abundances summarized by [7]. We let the iron abundance free for the fit to Suzaku data, and then fixed it, as well as the energies of the lines, to fit the other observations. As the lines are unresolved, the width of the lines was also fixed to $\sigma=0.1 \mathrm{eV}$. The normalization for Fe $\mathrm{K} \alpha$ and $\mathrm{K} \beta$ was fixed to the ratio given by the fluorescence yield, $Y_{K \beta} / Y_{K \alpha}=0.12537$ [8].

\footnotetext{
${ }^{1}$ http://pulsar.sternwarte.uni-erlangen.de/wilms/research/tbabs/
} 

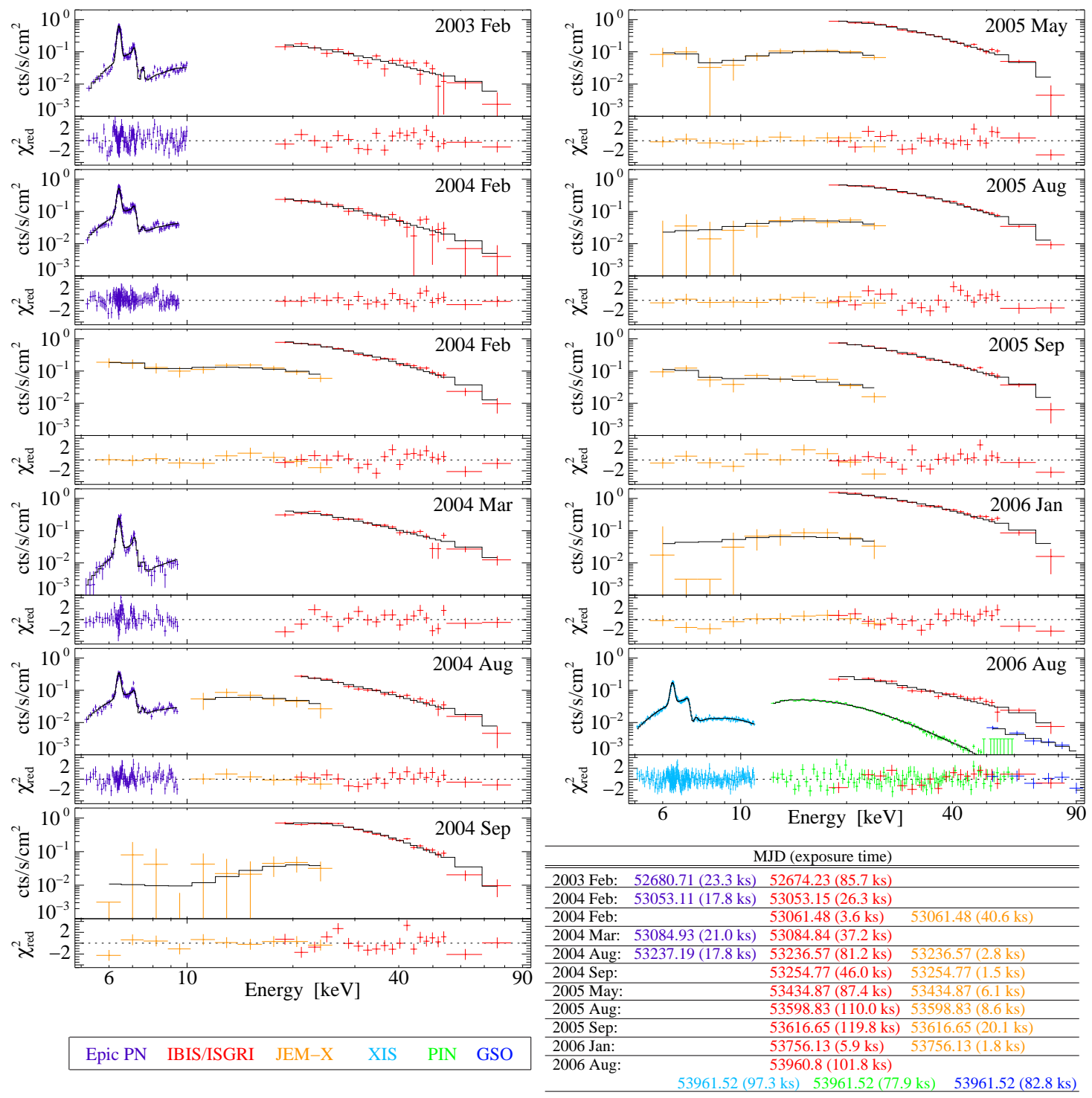

Figure 2: Spectra of all available XMM-Newton , JEM-X and Suzaku observations of IGR J16318-4848 together with the simultaneous data for IBIS/ISGRI. The model fitted is an absorbed cutoff powerlaw, with gaussians for the fluorescence lines (see text).

The spectrum below $5 \mathrm{keV}$ was ignored as it is most probably due to a source close to IGR J16318-4848 ([9, 10]).

Figure 3 shows the variation of the spectral parameters. Since the parameters are better constrained with XMM-Newton than with JEM-X, we will focus on the former. We can see that there is a change in the hydrogen column from $1.1(6) \times 10^{24} \mathrm{~cm}^{-2}$ to $3.4(7) \times 10^{24} \mathrm{~cm}^{-2}$. We did not detect a Compton shoulder for the $\mathrm{K} \alpha$ line at an upper limit for its flux of $1.8 \times 10^{-5} \mathrm{ph} \mathrm{cm}^{-2} \mathrm{~s}^{-1}$ [4]. These two facts are in agreement with the picture of the absorbing medium being the supergiant's highly inhomogeneous wind. The photon index and the cutoff energy are changing along the different observations as well, however, they are also consistent with a constant value within their uncertainties. 


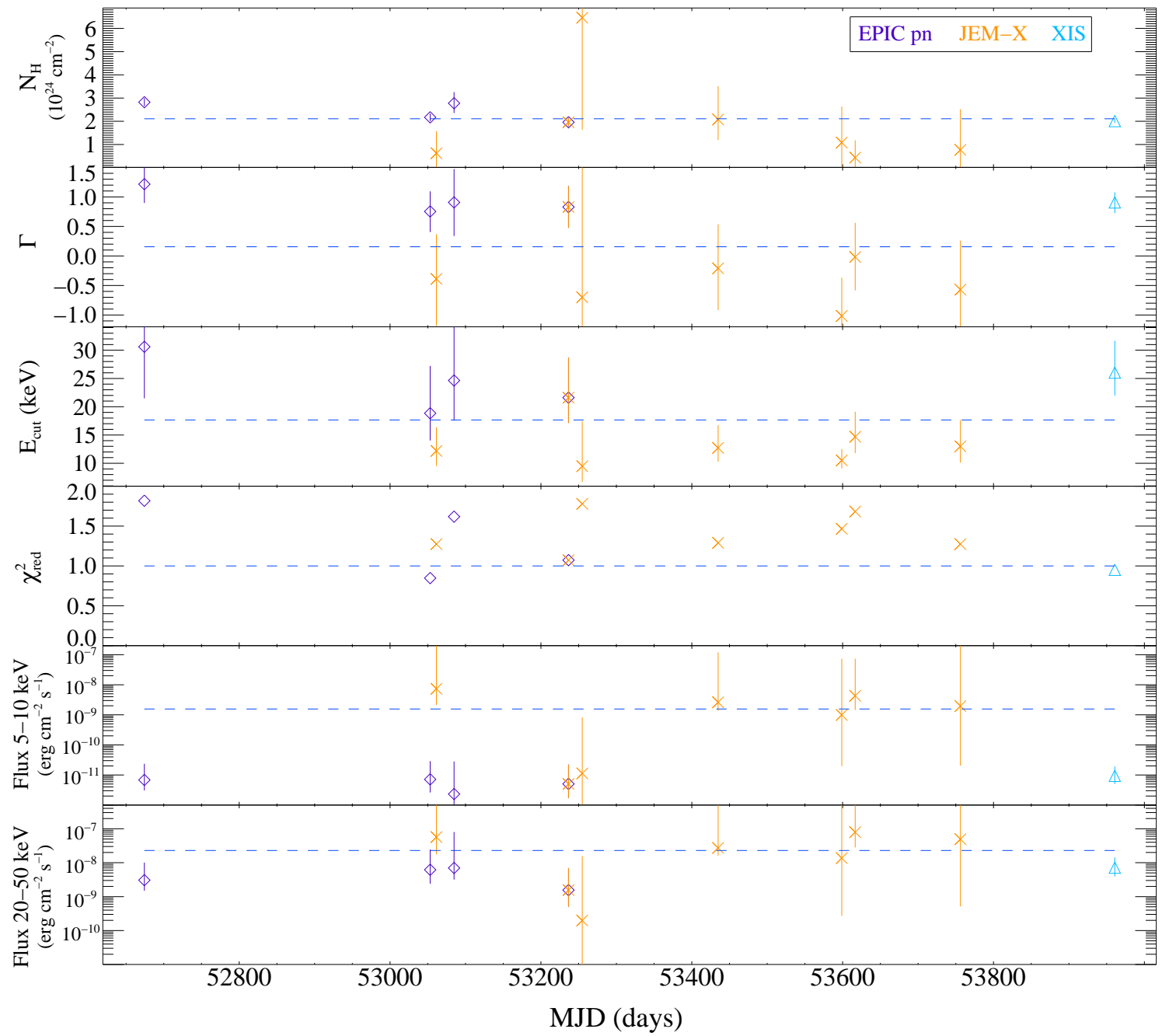

Figure 3: Parameters obtained from the fits to all available XMM-Newton, JEM-X and Suzaku observations together with IBIS/ISGRI (Fig. 2). The dashed lines show the (unweighted) mean of the corresponding values in each panel, except for the $\chi_{\text {red }}^{2}$, where the dashed line is at 1.0 to check for the goodness of the fit.

\section{Variability}

The light curve for the energy range $20-40 \mathrm{keV}$ of IBIS-ISGRI is shown in Fig. 4. The upper panel displays the total light curve with all the IBIS/ISGRI data available to us. The lower panels show some close-ups on the general light curve.

IGR J16318-4848 is varying significantly on short and long timescales. In the lower panels we can see how the source is constantly changing, with small short flares that are alternated with longer ones, like the one seen in the fourth lower panel. In these more intense flares, IGR J16318-4848 reaches more than 10 times its average count rate of $5.4 \mathrm{cps}$ in the $20-40 \mathrm{keV}$ band (IBIS/ISGRI).

This flaring behavior is typical for wind accreting systems, suggesting once more that IGR $\mathrm{J} 16318-4848$ is a system of a compact object imbedded in the dense wind of the $\mathrm{sgB}$ [e] star. 

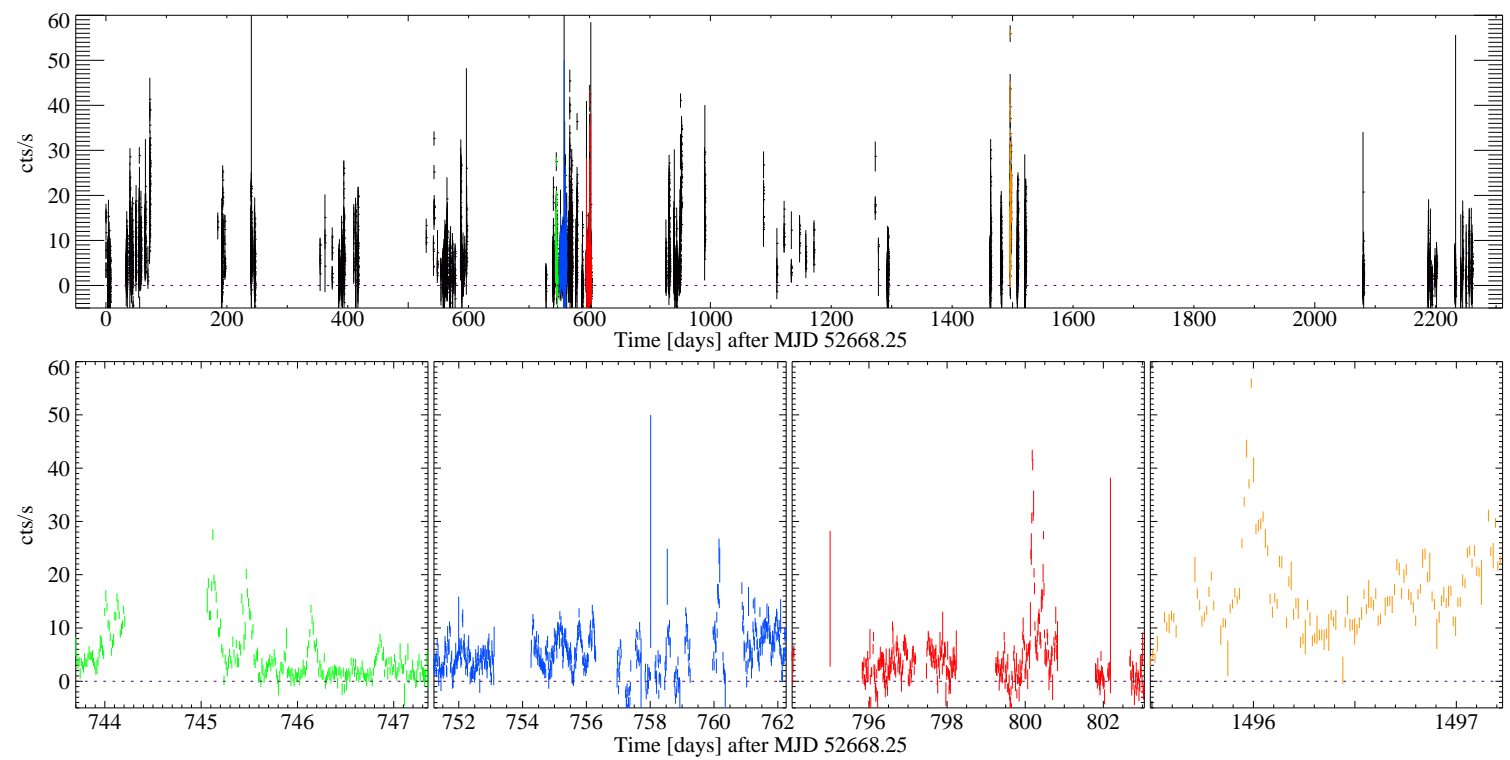

Figure 4: Upper pannel: IBIS/ISGRI light curve (20-40 keV) for all the archival data on IGR J16318-4848. Lower pannels: close-ups of different parts of the total light curve that show a flaring behavior typical of wind accreting systems (see text).

We were aiming to detect either a pulse period or an orbital period, thus we searched for a period from $1 \mathrm{~s}$ to $900 \mathrm{ks}$. We looked for any coherent oscillation in the light curve given by the events detected with the XIS and PIN onboard Suzaku, and also in a 75 s-binned light curve generated from all archival INTEGRAL data. We used the standard epoch folding for the search, but the result was negative and therefore we cannot determine the nature of the compact object in IGR J16318-4848.

\section{Outlook}

We will carry out a deeper analysis on the data, in particular we will:

- look for pulsations or the orbital period in the general light curve for IBIS/ISGRI with more sophisticated methods.

- study the fluorescence lines in detail: we will look for ionization changes in the long term (using the 5 XMM-Newton and Suzaku observations), and in the short term, dividing these observations into shorter intervals. A preliminar analysis showed us that we can still constrain the parameters for exposure times as short as $5 \mathrm{ks}$ each.

- analyze the IBIS/ISGRI observations (i.e. the observations where only this instrument is available), defining hardness ratios to look for spectral changes and compare those to actual changes in the continuum parameters provided by fits.

- compare the data from the satellites with Monte-Carlo simulations of the Compton scattering process.

- extend the analysis to other IGR sources, and compare the results obtained. 


\section{References}

[1] Filliatre P., Chaty S., 2004, ApJ 616, 469

[2] Revnivtesv M.G., 2003, Astronomy Letters 29, 644

[3] Walter R., Courvoisier T.J.L., Foschini L., et al., 2004, In: Schoenfelder V., Lichti G., Winkler C. (eds.) 5th INTEGRAL Workshop on the INTEGRAL Universe, Vol. 552. ESA Special Publication, p. 417

[4] Barragán L., Wilms J., Pottschmidt K., et al., 2009, A\&A 508, 1275

[5] Naik S., Paul B., 2004, A\&A 418, 655

[6] Hill A.B., Dean A.J., Landi R., et al, 2008, MNRAS 179

[7] Wilms J., Allen A., McCray R., 2000, ApJ 542, 914

[8] Kaastra, J. S. and Mewe, R., 1993, A\&AS 97, 443

[9] Ibarra A., Matt G., Guainazzi M., et al. 2007, A\&A 465, 501

[10] Matt G., Guainazzi M., 2003, MNRAS 341, L13 